\title{
Partial thromboplastin time test with kaolin: diagnosis of haemophilia and Christmas disease without natural reference plasmas
}

\author{
SUSAN F. KNIGHTS AND G. I. C. INGRAM \\ From the Department of Haematology, the Louis Jenner Laboratories, St. Thomas's Hospital and \\ Medical School, London
}

SYNOPSIS Deficiencies of factor VIII (in haemophilia) and factor IX (in Christmas disease) prolongo the partial thromboplastin time. If normal plasma is treated with alumina, the factor VIII remains? but the factor IX is removed and can subsequently be recovered by elution of the alumina. If a longpartial thromboplastin time is found on investigating a male patient whose history suggests a lifelong bleeding disorder, the plasma may be retested after adding either alumina-adsorbed normal plasma or eluate. If the patient's partial thromboplastin time is shortened (relative to the control) $\%$ by adding adsorbed normal plasma the patient is likely to be a haemophiliac; but if it is shortened:by adding eluate then he is likely to have Christmas disease. Practical details for carrying out theseo manoeuvres are given and experiments on the validity of the test described.

The partial thromboplastin time test with kaolin is a useful and simple test in the diagnosis of bleeding disorders. Abnormal results are obtained with deficiencies of those factors required for the formation of intrinsic thromboplastin, among them factors VIII and IX, so that the test may be used in screening for haemophilia or Christmas disease.

If a prolonged partial thromboplastin time is found on testing the plasma of a male with a lifelong bleeding disorder, and the prothrombin time is normal, the patient is likely to have either haemophilia or Christmas disease. A specific diagnosis may then be made in one of two ways. By one procedure, portions of the patient's and of a control plasma are added to plasmas from known cases of haemophilia and Christmas disease ('natural reference plasmas') and the degree of correction in the test is compared. Alternatively, materials rich in factors VIII or IX may be added to the patient's plasma and the degree of correction noted. The first method is of course the more specific, but the second can be performed in laboratories where natural reference plasmas are not available. Methods for the first procedure were given by Matchett and Ingram (1965), who also described in detail the partial thromboplastin time (P.T.T.) test using kaolin

Received for publication 4 January 1967. and Inosithin. This paper presents a method of work- $\stackrel{\mathbb{Q}}{\circ}$ ing the alternative procedure, and describes the $\overrightarrow{\vec{\sigma}}$ preparation of the factor-VIII- and IX-rich materials 3 from normal plasma.

\section{THE PROPOSED TEST}

The proposed test is based on the well-known observations that factor IX may be separated from factor VIII in plasma by adsorption onto alumina $\left(\mathrm{Al}(\mathrm{OH})_{3}\right)$ and subsequent elution. The test therefore consists in preparing $\mathrm{Al}(\mathrm{OH})_{3}$-treated normal plasma, and also an eluate $\mathrm{O}$ from the $\mathrm{Al}(\mathrm{OH})_{3}$ employed, and then performing P.T.T.s with and without these reagents added to the patient'so and the control plasma, and noting which additive has reduced the patient-control difference.

\section{MATERIAL AND METHODS}

MATERIALS The following are required.

$\mathrm{CaCl}_{2} \quad 0.025-\mathrm{M}$ solution in water

Trisodium citrate $3.2 \%$ solution in water

Tris buffer Aminotris(hydroxymethyl)methane buffer, $0.05-\mathrm{M}, p \mathrm{H} 7.4$ at $22^{\circ} \mathrm{C}$. (Gomori, 1946). Dissolve the tris base in water at a concentration of $2.43 \mathrm{~g} . \%$. Acidify $250 \mathrm{ml}$. of this solution with $42 \mathrm{ml}$. of $\mathrm{N}-\mathrm{HCl}$ and make $\mathrm{P}$ up to about $990 \mathrm{ml}$.; adjust the $p \mathrm{H}$ and make up to 1 litre. $\stackrel{\mathbb{Q}}{\Omega}$

Phosphate buffer at $\mathrm{pH} 8.0$ (Biggs and Macfarlane, 1962)

Dissolve (a) $1.118 \mathrm{~g}$. $\mathrm{Na}_{2} \mathrm{HPO}_{4} .2 \mathrm{H}_{2} \mathrm{O}$, and (b) 0.91 
$\mathrm{KH}_{2} \mathrm{PO}_{4}$, each in $100 \mathrm{ml}$. water; take $9 \cdot 7$ vol. of (a) with 0.3 vol. (b).

$\mathrm{Al}(\mathrm{OH})_{3}$ B.D.H. moist gel, diluted about 1 in 20 in tris buffer, so that when used to treat normal plasma as described under Methods, the prothrombin time is prolonged to $1 \cdot 5-4.0 \mathrm{~min}$.

Light kaolin Suspension, $5.0 \mathrm{mg} . / \mathrm{ml}$, in tris buffer.

Inosithin Soya bean extract forming a colloidal solution in a watery vehicle; dissolve in tris buffer at a concentration giving the shortest clotting time with normal plasma, about 1 to $2 \mathrm{mg}$./ml. (Associated Concentrates Inc., N.J.; obtainable through A.V. Howe and Co. Ltd., London).

METHODS The following preparations should preferably be carried out in plastic tubes. Mix $9.0 \mathrm{ml}$. normal blood with $1.0 \mathrm{ml}$. sodium citrate solution and centrifuge to obtain citrated plasma. Mix $4.5 \mathrm{ml}$. citrated plasma with $0.5 \mathrm{ml}$. Al(OH) $)_{3}$-suspension; incubate at $37^{\circ} \mathrm{C}$. for $3 \mathrm{~min}$. and centrifuge. Decant the adsorbed plasma and confirm that the prothrombin time does not exceed $4 \mathrm{~min}$. (or factor-VIII activity may be lost). Dilute 1 in 5 by adding $0.5 \mathrm{ml}$. to $2.0 \mathrm{ml}$. tris buffer: this is referred to as 'adsorbed plasma 1 in 5 '. Wash the deposited $\mathrm{Al}(\mathrm{OH})_{3}$ three times by resuspension in about $5.0 \mathrm{ml}$. chilled distilled water and centrifugation, and then resuspend in $0.45 \mathrm{ml}$. phosphate buffer, i.e., $1 / 10$ th the original plasma volume, incubate $5 \mathrm{~min}$. at $37^{\circ} \mathrm{C}$., and centrifuge. Dilute the supernatant 1 in 8 by adding $0.2 \mathrm{ml}$. to $1.4 \mathrm{ml}$. tris buffer (to dilute out the inhibitory effect of the phosphate buffer): this is referred to as 'eluate'.

To perform the test, set up partial thromboplastin time tests on the patient's and the control plasma as follows. Into six clotting tubes (which may be glass or plastic) in the waterbath at $37^{\circ} \mathrm{C}$. place $0.1 \mathrm{ml}$. kaolin suspension and $0.1 \mathrm{ml}$. Inosithin. To the first tube add $0.1 \mathrm{ml}$. control plasma, tap the tube to mix the contents, and start a watch. One minute later, add $0.1 \mathrm{ml}$. patient's plasma to the second tube and mix. At successive minutes similarly add $0.1 \mathrm{ml}$. control or patient's plasma alternately to the remaining tubes. As the watch approaches $10 \mathrm{~min}$., complete the reaction mixture in the first tube by adding $0.1 \mathrm{ml}$. tris buffer and, at exactly $10 \mathrm{~min} ., 0.1 \mathrm{ml}$. $\mathrm{CaCl}_{2}$ solution, and time the clotting on another watch. At 11 min., similarly complete the mixture in the second tube with buffer and $\mathrm{CaCl}_{2}$ solution, and time the clotting. In the same way, at 12 and $13 \mathrm{~min}$. add adsorbed plasma 1 in 5 instead of buffer to the next pair of tubes, and at 14 and $15 \mathrm{~min}$. add eluate to the final pair of tubes, and recalcify. The longer clotting times will overlap each other but this does not matter. The test is only informative if big differences are established between the effects of adding adsorbed plasma 1 in 5 and eluate, so that the endpoints need not be determined with great precision.

Repeat the whole manoeuvre a second time in the opposite order to exclude a temporal drift due to changes in the reagents, and calculate the means of the duplicates. From these, tabulate the difference patient's time minus control time for each of the additions (buffer, adsorbed plasma 1 in 5 , and eluate).

Interpretation In factor-VIII deficiency, the difference tabulated for adsorbed plasma 1 in 5 will be less than the other two, whereas with factor-IX deficiency the eluate difference will be less than the other two. (Such differences between differences are described below as 'second-degree differences'.)

Clearly, the greater the difference with tris, the more will it be reduced by the additive containing the missing factor; so that severe deficiencies will be more clearly identified than those which are mild; in fact the procedure will usually fail to discriminate when the initial patientcontrol difference is less than 10 seconds. The test will also fail if the patient's plasma contains a rapid inactivator of the deficient factor.

\section{EXPERIMENTS ON THE VALIDITY OF THE PROPOSED TEST}

THE EFFECT OF SERUM ON HAEMOPHILIC PLASMA It was wrongly stated by Matchett and Ingram (1965) that the addition of serum to the partial thromboplastin time test would distinguish between deficiencies of factors VIII and IX. In fact, serum will sometimes shorten the long partial thromboplastin time of haemophilic plasma about as much as adsorbed plasma (Table I). Adding eluate, however, does not shorten the test so much as adding serum (Table II), and thus gives a clearer comparison with the effect of adsorbed plasma.

\section{TABLE I}

RESULTS OF THE ADDITION OF ADSORBED PLASMA OR SERUM TO HAEMOPHILIC PLASMA

\begin{tabular}{lcll} 
Plasma & \multicolumn{2}{l}{ Addition } \\
\cline { 2 - 4 } & None & $\begin{array}{l}\text { Diluted Adsorbed } \\
\text { Plasma }\end{array}$ & Diluted Serum \\
\hline Haemophiliac 1 & 147 & 74 & 81 \\
Control & 42 & 44 & 38 \\
$\quad$ Difference & 105 & 30 & 43 \\
Haemophiliac 2 & 128 & 89 & 82 \\
Control & 39 & 42 & 35 \\
$\quad$ Difference & 89 & 47 & 47
\end{tabular}

Partial thromboplastin time tests on the plasmas of two haemophiliacs and their controls, and with the addition (to both) of diluted adsorbed normal plasma and diluted aged normal serum (in the first case the additions were diluted 1 in 5 , and in the second case 1 in 10). The addition of serum shortens the test as much as the addition of adsorbed plasma and so does not discriminate haemophilia from Christmas disease.

Each entry is the mean of two readings.

TEST WITH CHRISTMAS PLASMA Table III shows that, with Christmas disease plasma, the partial thromboplastin time is shortened by the addition of eluate but not by adding adsorbed plasma.

RELATION TO TESTS WITH NATURAL REFERENCE PLASMAS It was previously suggested that a 1 in 20 dilution of the patient's and the control plasmas in 
TABLE II

RESULTS OF ADDITION OF BUFFER, ADSORBED PLASMA, SERUM, OR ELUATE TO HAEMOPHILIC AND CONTROL PLASMAS

Plasma Addition

\begin{tabular}{llll}
\hline Buffer & $\begin{array}{l}\text { Adsorbed } \\
\text { Plasma } 1 \text { in } 5\end{array}$ & Serum 1 in 5 & Eluate \\
\hline
\end{tabular}

\begin{tabular}{lrrrl}
\hline 1 & 63 & 20 & 42 & 67 \\
2 & 30 & 15 & 23 & 31 \\
3 & 70 & 12 & 58 & 67 \\
4 & 39 & 13 & 29 & 34 \\
5 & 116 & 32 & 48 & 66 \\
6 & 53 & 15 & 40 & 45 \\
Mean & 61 & 18 & 40 & 52
\end{tabular}

Haemophilic-control differences (sec.) in the partial thromboplastin time test with the additions (to both plasmas) shown. The differences with eluate were consistently greater than with serum; eluate only slightly shortened the partial prothrombin times: $C f$. the differences with buffer.

Each entry is derived from the means of two readings.

TABLE III

RESULTS OF THE ADDITION OF ADSORBED

PLASMA OR ELUATE TO CHRISTMAS DISEASE AND CONTROL PLASMAS

Plasma Addition

Buffer Adsorbed Plasma 1 in 5 Eluate

$\begin{array}{lll}78 & 91 & 26\end{array}$

$\begin{array}{lll}45 & 54 & 25 \\ 57 & 82 & 34\end{array}$

3 Christmas-control differences (sec.) in the partial thromboplastin time test with the additions (to both plasmas) shown. With each plasma, eluate shortened the test whereas adsorbed plasma somewhat lengthened it. There is an obvious distinction from the results of Table II. Each entry is derived from the means of two reading 3 .

natural reference plasmas would provide a simple and sensitive specific test for deficiencies of factors VIII or IX (Matchett and Ingram, 1965). The sensitivity of the present procedure in detecting factor-VIII deficiency was therefore compared with the 1-in-20 test using haemophilic plasma. A series of mixtures of normal plasma in haemophilic plasma were prepared up to a dilution of 1 in 50 of the normal plasma, to represent various degrees of haemophilic detect. Each dilution, and the undiluted normal plasma, were then tested in the partial thromboplastin tıme test at 1 in 20 in haemophilic plasma. The differences were then calculated between the clotting times obtained from the undiluted plasma and from each of the plasma mixtures. Similarly, the undiluted normal plasma and the mixtures with haemophilic plasma were tested by the present procedure, adding, in turn, tris, and adsorbed plasma 1 in 5 , to each. Here, the differences in clotting time were calculated between each mixture and the undiluted plasma, for both additions; and then the differences with adsorbed plasma were subtracted from the corresponding differences with tris to give

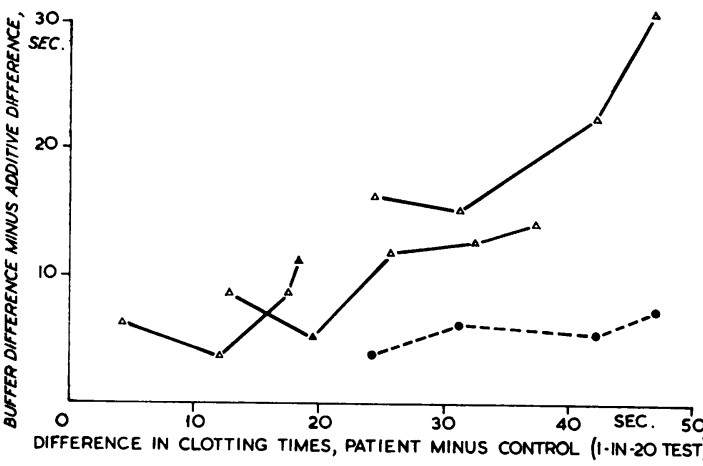

FIG. 1. Test-control difference for the present test plotted against those from the 1-in-20 procedure, for artificial mixtures of normal plasma in haemophilic plasma.

$\triangle$ addition of adsorbed plasma. addition of eluate The effects of adding adsorbed plasma roughly correspond to the results of the 1-in-20 test; the addition of eluate produces only minimal shortening irrespective of the degree of haemophilic defect.

Each point is derived from the means of two readings.

the net effect of adsorbed plasma in the various mixtures, that is, the degree of correction of the haemophilic defect.

In Fig. 1, these second-degree differences from the present test are plotted against the 1-in-20 differences, to show the relative sensitivity of the two tests to mild factor-VIII deficiency. The 1-in-20 procedure appears to be the more sensitive, in that differences with this test in the $10-20 \mathrm{sec}$. range correspond to differences of less than $10 \mathrm{sec}$. with the present test. With more marked differences (corresponding to a more severe deficiency of factor VIII), the tests have a similar sensitivity.

For comparison, data were also obtained in one of the experiments by adding eluate instead of adsorbed plasma, and these are included in Figure 1. The results confirm those shown in Table II, that the effect of adding eluate to haemophilic plasma is negligible; for the difference with eluate is hardly less than the difference after adding buffer, and is $\tilde{N}$ independent of the degree of haemophilic defect.

RELATION TO THE DEGREE OF HAEMOPHILIC DEFECT In the present context, the simplest measure of the severity of factor-VIII deficiency is the degree of prolongation of the partial thromboplastin time. In Fig. 2 is $\stackrel{\varnothing}{\oplus}$ therefore plotted the second-degree difference representing the net effect of adsorbed plasma in the present test against the test-control difference from the ordinary partial thromboplastin time. In $\frac{?}{\mathbb{D}}$ general there is seen to be a good correlation between the two readings, showing that the more severe the haemophilic defect, the more obvious will $\delta$ 


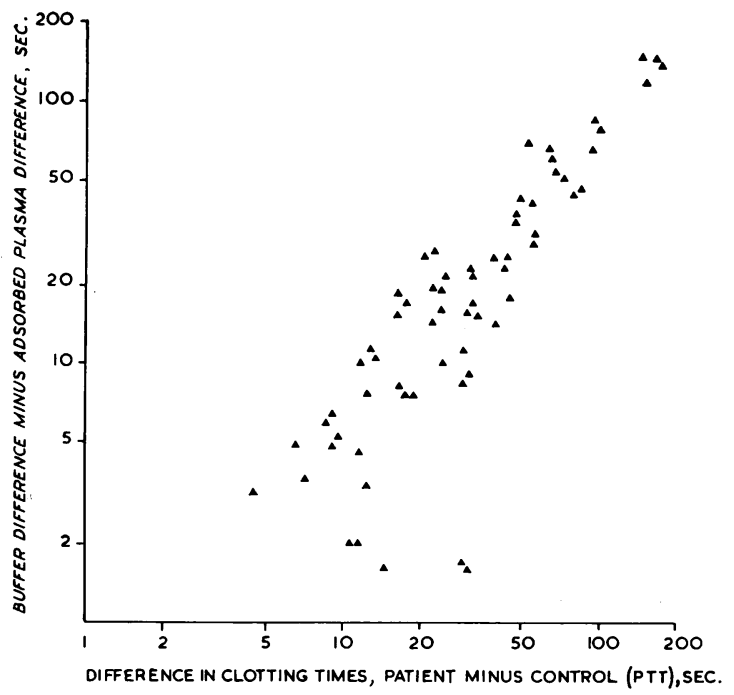

FIG. 2. Results on haemophilic plasmas. Patient-control differences from the present test after adding adsorbed plasma, plotted against those from ordinary partial thromboplastin times (log. scales).

The range of values was obtained by testing samples from haemophiliacs before and at various intervals after infusions of factor-VIII-containing materials. The aberrant points are commented on in the text. Each point is derived from the means of two readings.

be the correction with adsorbed plasma in the present test. Figure 2, however, shows two obviously aberrant readings. In these determinations there was a marked drift shown by increasing differences between the first and second replicate readings as the test proceded, an example of the possibility referred to under Methods. It would be wise to discard readings which show this phenomenon and repeat the test.

COMPARISON BETWEEN THE RESULTS OF THE TEST ON HAEMOPHILIC AND CHRISTMAS DISEASE PLASMA A series of tests was carried out on both haemophilic and Christmas disease plasmas, adding, in turn, to each, tris, adsorbed plasma, and eluate. Figure 3 shows the difference between the results on adding tris and either adsorbed plasma 1 in 5 or eluate, whichever difference was the larger, plotted against the patient-control difference with tris alone. It is clear that Christmas disease plasma is corrected by eluate in a closely similar way to the correction of haemophilic plasma by adsorbed normal plasma. Inspection of the other results, i.e., the effect of eluate on haemophilic plasma, and the effect of adsorbed plasma on Christmas disease plasma, showed no evidence of correction, confirming the results of Table III.

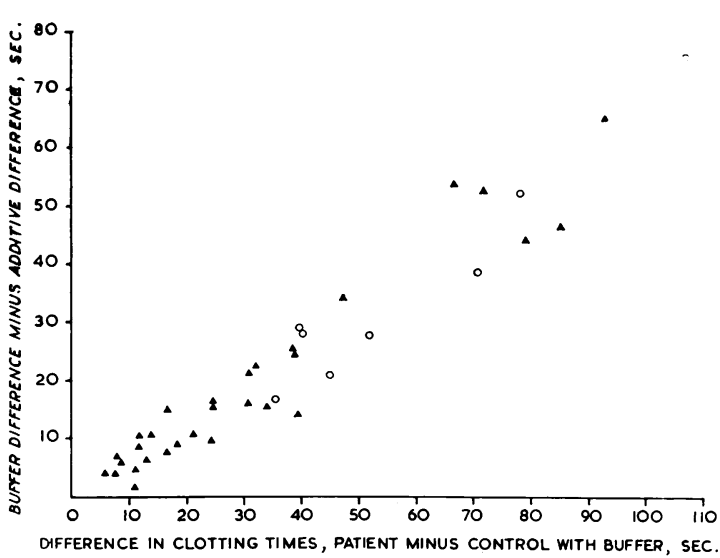

FIG. 3. Results on haemophilic and Christmas disease plasmas. Second-degree differences from haemophilic plasma with adsorbed plasma 1 in 5 , and from Christmasdisease plasma with eluate, plotted against the patientcontrol difference with buffer.

$\triangle$ haemophilic plasma with adsorbed plasma 1 in 5.

$\bigcirc$ Christmas-disease plasma and eluate.

The data show that the procedures suggested for haemophilic and Christmas-disease plasma have a similar sensitivity. Each point is derived from the means of two readings.

\section{DISCUSSION}

In the experiments reported above, we have worked with known haemophilic and Christmas-disease plasmas. In field tests with unknown clinical samples, the procedures are of course not specific for deficiencies of factor VIII and factor IX but have the same inferential specificity as the thromboplastin generation test (Biggs and Douglas, 1953). The 'cross-over' tests with control adsorbed plasma and patients' serum, and patients' adsorbed plasma and control serum in the thromboplastin generation test correspond to the addition of normal adsorbed plasma 1 in 5 and of eluate in our procedure, and in both cases the diagnosis of haemophilia or of Christmas disease (according to which combination 'corrects' the test) is based on probabilities. By contrast the 1-in-20 procedure of Matchett and Ingram (1965) is specific because it is based on natural reference plasmas with known unique deficiencies. The relative frequencies of the different bleeding disorders in human populations, however, allows the inferential diagnoses of haemophilia or Christmas disease to be made with sufficient confidence to be of clinical value, as the widespread use of the thromboplastin generation test has shown.

\section{REFERENCES}

Biggs, R., and Macfarlane, R. G. (1962). Human Blood Coagulation and its Disorders, 3rd, ed. p. 369. Blackwell, Oxford.

$\longrightarrow$, and Douglas, A. S. (1953). J. clin. Path., 6, 23.

Gomori, G. (1946). Proc. Soc. exp. Biol. (N.Y.), 62, 33

Matchett, M. O., and Ingram, G. I. C. (1965). J. clin. Path., 18, 465. 